\title{
SOUTHERN REFLECTION NEBULAE
}

\author{
SIDNEY VAN DEN BERGH and RENÉ RACINE \\ David Dunlap Observatory, University of Toronto, Canada
}

\begin{abstract}
The Curtis Schmidt telescope at the Cerro Tololo Observatory has been used for a twocolor survey of reflection nebulae in the Southern Milky Way. Major concentrations of reflection nebulae are found in Carina and in the zone $250^{\circ}<l^{11}<270^{\circ}$. A conspicuous gap in the distribution of reflection nebulae occurs at $l^{\mathrm{II}} \simeq 275^{\circ}$. A detailed photometric and spectroscopic investigation of the stars imbedded in southern reflection nebulae is now in progress.
\end{abstract}

\section{Introduction}

In previous papers (van den Bergh, 1966; Racine, 1968) it has been shown that stars which are imbedded in reflection nebulae occur in associations. Typically the stars in such $\mathrm{R}$ associations are $\sim 100$ times brighter than the unstable main sequence dwarfs that populate $T$ associations. $R$ associations can therefore be studied conveniently out to considerably greater distances than $T$ associations.

Racine (1968) and Racine and van den Bergh (1970) were able to show that $R$ associations outline the Orion spiral arm rather better than do $O B$ associations. The reason for this is that the number of $\mathrm{R}$ associations per $\mathrm{kpc}^{2}$ in the galactic plane is greater than the number of $\mathrm{OB}$ associations. This is so because most $\mathrm{R}$ associations consist of late B- and early A-type stars which have a much greater space density than do the $\mathrm{O}$ - and early B-type stars that outline $\mathrm{OB}$ associations.

\section{The Southern Survey}

The interpretation of the optical spiral arm pattern in the vicinity of the sun is still the subject of considerable controversy (Bok, 1970). The true nature of the spiral pattern is particularly uncertain in the southern sky. It therefore seemed worthwhile to extend the northern reflection nebula survey (that had been carried out on the prints of the Palomar Sky Survey) to the southern hemisphere. A strip with an average width $\sim 12^{\circ}$ was therefore photographed in two colors with the 24 -in. $(61-\mathrm{cm})$ Curtis Schmidt Telescope at the Cerro Tololo Observatory in Chile. The plates for this southern survey were taken in 1969, 1970 and 1971. The blue exposures were mostly on IIa-O emulsion behind a GG13 filter; the red exposures on 098-02 emulsion behind and RG2 filter. Most of the plates were developed in MWP 2.

Compared to the 48-in. $(126-\mathrm{cm})$ Palomar Schmidt the Curtis telescope has two disadvantages:

(1) Faint nebulosity is more easily detected on plates taken with the $f / 2.5$ Palomar instrument than on plates taken with the $f / 3.5$ Curtis telescope. 
(2) The large size and complex structure of the brighter stellar images produced by the Curtis Schmidt makes it much more difficult to detect small reflection nebulae around bright stars on Tololo plates than on Palomar plates.

\section{Discussion}

Approximately 100 stars embedded in reflection nebulae were found during the course of the present survey (to guard against plate defects only those nebulae that could be seen on both blue and red plates were catalogued). The distribution of these objects is plotted in Figure 1. Also plotted in this figure are the reflection nebulae that had previously been found on the Sky Survey prints of the northern sky.

Figure 1 shows that the southern reflection nebulae between $250^{\circ}<l^{\mathrm{II}}<350^{\circ}$ are located much closer to the galactic plane than are those that had previously been found on the Palomar Sky Survey prints. The reasons for this are the following:
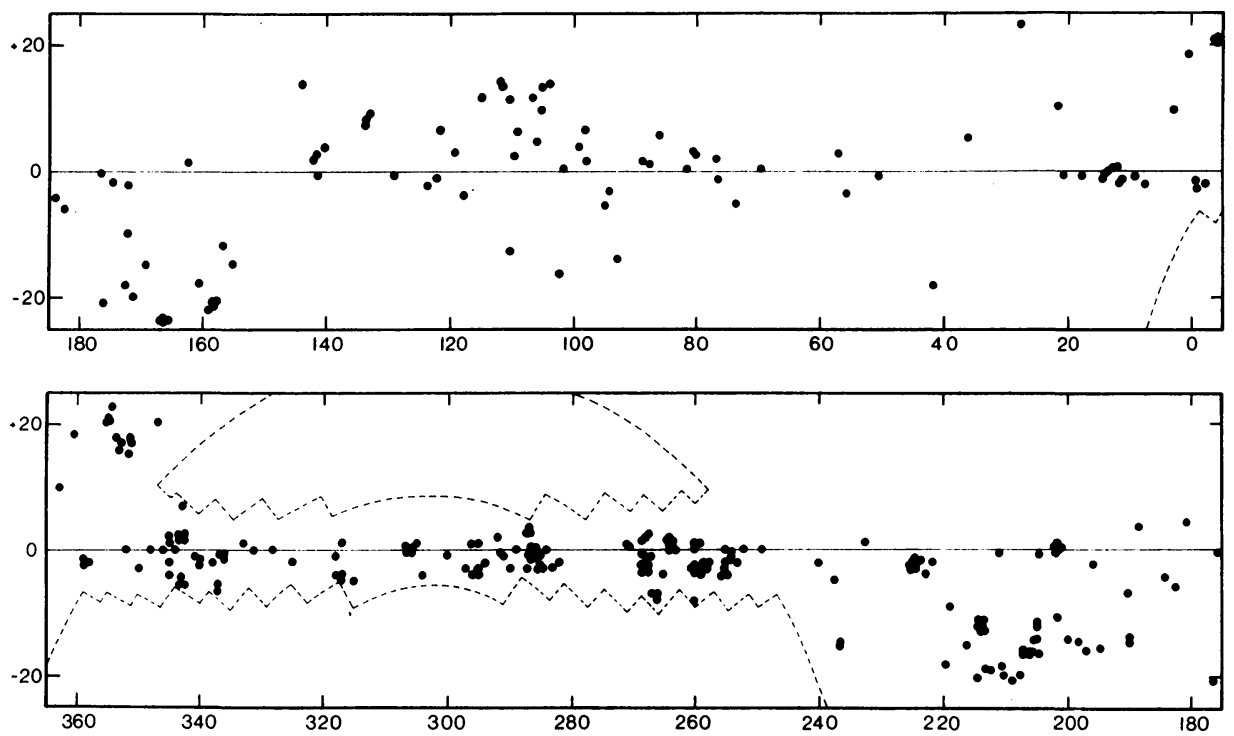

Fig. 1. Distribution of stars in reflection nebulae in the northern (top) and southern (bottom) Milky Way. Note the very clumpy distribution of reflection nebulae. Dotted lines show the limits of the present survey.

(1) The southern survey only extends to $\left|b^{\mathrm{II}}\right| \simeq 6^{\circ}$.

(2) The southern survey includes all stars in reflection nebulae whereas the northern survey was confined to BD stars. On the average the southern stars in reflection nebulae are therefore fainter and hence more distant than their northern counterparts.

(3) The southern survey covers a region in which 'Gould's Belt' crosses the galactic equator.

Inspection of Figure 1 shows that the distribution of southern reflection nebulae is quite clumpy. Major concentrations of nebulae are found in Carina and in the zone 
$250^{\circ}<l^{\mathrm{II}}<270^{\circ}$. A conspicuous gap in the distribution of reflection nebulae occurs at $l^{\mathrm{II}} \approx 275^{\circ}$.

$\mathrm{Mr}$ Bill Herbst has recently used the University of Toronto $24-i n .(61-\mathrm{cm})$ reflector on Las Campanas in Chile to obtain classification spectra at a dispersion of $128 \AA$ $\mathrm{mm}^{-1}$ of all southern stars with $B<10$ that are located in reflection nebulae. Spectra of fainter stars will be obtained with an image tube spectrograph. UBV photometry of some of the faint stars in reflection nebulae has been obtained with the Cerro Tololo 60-in. (1.5-m) telescope. Additional UBV observations are planned with the Toronto 24 -in. $(61-\mathrm{cm})$ telescope in Chile. Many of the stars in reflection nebulae appear to be members of small clusters. Photometric observations of the stars in these clusters are also planned.

The photometric and spectroscopic observations outlined above will be used to determine the distances to southern associations of reflection nebulae. It is hoped that the combined data on the distances of northern and southern reflection nebulae will lead to an improved picture of nearby spiral structure.

\section{Acknowledgements}

We are deeply grateful to Dr Victor Blanco for making large blocks of observing time on the Curtis Schmidt telescope available to us for this project. We also thank Steven van Agt, Tom Barnes, Chris Coutts, and Barry Madore for contributing to the Southern Milky Way Survey. This investigation was supported by the National Research Council of Canada.

\section{References}

Bergh, S. van den: 1966, Astron. J. 71, 990.

Bok, B. J.: 1970, Sky Telescope 39, 21.

Racine, R.: 1968, Astron. J. 73, 233.

Racine, R. and Bergh, S. van den: 1970, in W. Becker and G. Contopoulos (eds.), 'The Spiral Structure of Our Galaxy', IAU Symp. 38, 219. 